OPEN ACCESS

Edited by:

Aiping Lu,

Hong Kong Baptist University,

Hong Kong

Reviewed by:

Yu Huang,

The Chinese University of Hong Kong,

China

Lei Chen,

Fujian Agriculture and Forestry

University, China

${ }^{*}$ Correspondence:

Yu-ling Ma

yu-ling.ma@dpag.ox.ac.uk

Specialty section:

This article was submitted to

Ethnopharmacology,

a section of the journal

Frontiers in Pharmacology

Received: 22 August 2018

Accepted: 21 January 2019

Published: 06 February 2019

Citation:

Wang T, Xie W, Yu J, Ellory C.

Wilkins R, Zhu Y and Ma Y-I (2019) Ion

Channel Targeted Mechanisms

of Anti-arrhythmic Chinese Herbal

Medicine Xin Su Ning.

Front. Pharmacol. 10:70.

doi: 10.3389/fphar.2019.00070

\section{Ion Channel Targeted Mechanisms of Anti-arrhythmic Chinese Herbal Medicine Xin Su Ning}

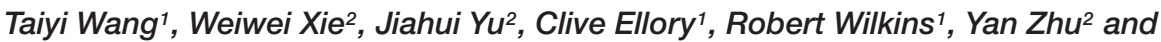 \\ Yu-ling Ma ${ }^{1 *}$ \\ ${ }^{1}$ Department of Physiology, Anatomy and Genetics, University of Oxford, Oxford, United Kingdom, ${ }^{2}$ Tianjin State Key \\ Laboratory of Modern Chinese Medicine, Tianjin University of Traditional Chinese Medicine, Tianjin, China
}

Xin Su Ning (XSN) is a China patented and certified herbal medicine used to treat premature ventricular contractions (PVCs) since 2005. A recent completed clinical trial of 861 patients showed that XSN had similar PVC inhibition rate to the class I antiarrhythmic drug mexiletine, at $65.85 \%$ for XSN and $63.10 \%$ for mexiletine. We have previously reported that XSN prolongs action potential duration (APD) and suppresses action potential amplitude (APA) of the cardiac ventricular myocytes. In this report we aim to reveal the effect of XSN on the ionic channels that govern APD and APA, which would help to explain the cellular electrophysiological mechanism of XSN. Our main findings are: (1) On ECG recorded in isolated rat, in the presence of XSN the amplitude of $R$ wave was significantly decreased and the amplitude of $T$ wave was increased significantly; (2) XSN blocked hNaV1.5 channel stably transfected cell line in a dose-dependent manner with an $\mathrm{IC}_{50}$ of $0.18 \pm 0.02 \mathrm{~g} / \mathrm{L}$; and (3) XSN suppresses hERG channels in a dose-dependent manner with an $I_{50}$ of $0.34 \pm 0.01 \mathrm{~g} / \mathrm{L}$. In conclusion, the clinical antiarrhythmic efficacy of XSN is based on its class I and Class III antiarrhythmic properties by suppression hNaV1.5 channel and hERG channels, which are directly responsible for XSN's effect on APA suppression and APD prolongation.

Keywords: anti-arrhythmic drugs, premature ventricular contractions, Xin Su Ning, Chinese Herbal Medicine, electrophysiology

\section{INTRODUCTION}

Although much research has been carried out in the attempt to gain better understanding of cardiac arrhythmia, and in the search for effective and safe drugs to treat this common cardiac condition, the progress has been not very encouraging due to the multifactor and dynamic nature of the disease's causes and its development (Rosen, 1988; Weiss et al., 2015). New drug development strategy of targeting ion channels or cellular electrophysiological properties have developed sophistically in the last decades, however the drugs discovered in this scheme are far from satisfactory, due to adverse reactions of the antiarrhythmic drugs (Williams, 1992; Ma et al., 2006; Frommeyer and Eckardt, 2016; O'rourke et al., 2016; Sun et al., 2016). In this report we aimed to study the antiarrhythmic mechanism of a clinically effective multi-herbal/multicomponent Chinese medicine XSN, which we hope to elicit the cellular electrophysiological property of XSN and to provide guidance for clinicians who use XSN to treat cardiac arrhythmia patients, we also hope to open up a research field to understand the antiarrhythmic actions of multicomponent medicine. 
Xin Su Ning (XSN) is a multi-herbal medicine patented and certified to produce in China. It has been available in China since 2005 for treating cardiac ventricular arrhythmia, especially arrhythmias induced by cardiac ischemia and viral myocarditis (Zhai et al., 2017). XSN is comprised of 11 herbs: Coptidis Rhizoma (Huanglian, Coptis chinensis Franch.), Pinelliae Rhizoma [Banxia, Pinellia ternata (Thunb.) Makino], Poria [Fuling, Poria cocos (Schw.) Wolf], Aurantii Fructus Immaturus (Zhishi, Citrus aurantium L.), Dichroae Radix (Changshan, Dichroa febrifuga Lour.), Nelumbinis Plumula (Lianzixin, Nelumbo nucifera Gaertn.), Sophorae Flavescentis Radix (Kushen, Sophora flavescens Ait.), Artemisiae Annuae Herba (Qinghao, Artemisia annua L.), Ginseng Radix et Rhizoma (Renshen, Panax ginseng C. A. Mey.), Ophiopogonis Radix (Maidong, Ophiopogon japonicus (L. f) Ker Gawl.), and Nardostachyos Radix et Rhizoma (Gancao, Glycyrrhiza uralensis Fisch.).

The pre-licensing pharmacological studies in China showed that XSN significantly suppressed cardiac arrhythmia induced by the chemical reagents Matrin, $\mathrm{CaCl}_{2}, \mathrm{Chloroform}$, and Isoproterenol. In cardiac ischemia-induced arrhythmia, XSN significantly delayed the onset of ventricular arrhythmia and shortened the time of arrhythmia. The pre-licensing toxicity study showed that XSN did not induce any abnormal changes to blood and urine, and the application of XSN did not cause any toxic reactions in the heart, the liver or the kidney. A recent clinical study of 861patients showed that the inhibition rate of the PVC by XSN was similar to that of mexiletine, at $65.85 \%$ for XSN and $63.10 \%$ for mexiletine (Zhai et al., 2017). An earlier clinical study reported that XSN significantly improved the overall symptoms of patients with tachycardia-associated conditions compared with propafenone $(P<0.05)$ (Yuan and Zhou, 2000). In addition, since XSN was licensed for use clinically to treat cardiac arrhythmias, there have been no adverse reactions induced by XSN being reported.

With increasing popularity of the complementary and alternative medicine among patients with PVCs, TCM (Traditional Chinese Medicine) has been more frequently used both in China and in the western countries (Zou et al., 2011; Hua et al., 2015; Wang et al., 2016). There are other antiarrhythmic Chinese medicine available in China, such as Wenxin Keli (WK) and Shen Song Yang Xin Capsule (SSYX). It was reported that WK has cardio-protective and antiarrhythmic properties with the regulatory actions on ion channels, such as $\mathrm{I}_{\mathrm{Na}}, \mathrm{I}_{\mathrm{Na}} \mathrm{L}, \mathrm{I}_{\mathrm{to}}, \mathrm{I}_{\mathrm{CaL}}$, and $\mathrm{I}_{\mathrm{f}}$ (Burashnikov et al., 2012; Tian et al., 2018). The other well-known antiarrhythmic TCM is SSYX. It was reported that SSYX blocks various ion channels such as $\mathrm{I}_{\mathrm{Na}}, \mathrm{I}_{\mathrm{CaL}}, \mathrm{I}_{\text {to }}$, and $\mathrm{I}_{\mathrm{K} 1}$ (Li et al., 2007; Zou et al., 2011).

Our previous study showed that XSN prolongs APD, a class III anti-arrhythmic characteristics in isolated cardiac myocytes (Ma et al., 2017). We report here the effect of XSN on ECG of isolated hearts, on hNav1.5 sodium channel and hERG potassium channel to illustrate a picture of the electrophysiological mechanism and to understand how XSN exerts its antiarrhythmic action clinically.

\section{MATERIALS AND METHODS}

\section{Medicine and Chemicals}

XSN was provided by Shaanxi Momentum Pharmaceutical Co., Ltd. (Shaanxi, China) as an frozen dried powder of the extract of the 11 herbs described above (detailed preparation procedures is described in the Supplementary Material). The chemical composition of XSN was studied and has been reported recently by Guo et al. (2018), using the ultra-high-pressure liquid chromatography coupled with linear ion trap-Orbitrap tandem mass spectrometry (UHPLC-LTQ-Orbitrap), 41 components were identified as candidate bioactive components. All other chemicals were purchased from Sigma-Aldrich (Gillingham, Dorset, United Kingdom) and used as supplied unless stated.

\section{The Animals Used}

Male Sprague-Dawley rats (280-320 g) were used for the isolated heart perfusion experiments. The rodents were housed in cages at a temperature of $22^{\circ} \mathrm{C} \pm 2{ }^{\circ} \mathrm{C}$ and humidity $40 \% \pm 5 \%$, under a $12 \mathrm{~h}$ light/dark cycle, and received standard diet and water ad libitum. All experiments were reviewed and approved by the Committee of Ethics on Animal Experiments at the TJAB (TJAB-JY-2011-002) and were carried out under the Guidelines for Animal Experiments at the Tianjin University of Traditional Chinese Medicine.

Male Wistar rats, weighing 200-250 g were used in primary cardiomyocytes isolation and were given standard laboratory chow and water ad libitum. All investigations conformed to the Guide for the Care and Use of Laboratory Animals published by the US National Institutes of Health (NIH Publication No. 8523, revised 1996), the Home Office Guidance on the Operation of the Animals (Scientific Procedures) Act, 1986 (HMSO), and to institutional guidelines. Approval was granted by the University of Oxford Animal Ethics Review Committees and the Home Office (Project License numbers 30/2278 and 30/2755).

\section{ECG Recording}

Prior the study of the effect of XSN on ion channels we carried out a group of experiment to test the effect of XSN on ECG of isolated Rat hearts. We selected a concentration that is $1 / 2$ of IC50 of the APD prolongation of $\mathrm{XSN} \sim 0.8 \mathrm{~g} / \mathrm{l}$ in rat ventricular myocytes. The SD rats were anesthetized with isoflurane, the hearts were excised and immediately immersed in cold Krebs-Henseleit $(\mathrm{KH})$ solution contained the following $(120 \mathrm{mM} \mathrm{NaCl}, 4.7 \mathrm{mM}$ $\mathrm{KCl}, 25.0 \mathrm{mM} \mathrm{NaHCO}, 1.2 \mathrm{mM} \mathrm{KH}{ }_{2} \mathrm{PO}_{4}, 1.2 \mathrm{mM} \mathrm{MgSO}$, $11.1 \mathrm{mM}$ glucose, $2.0 \mathrm{mM}$ Na-pyruvate and $1.8 \mathrm{mM} \mathrm{CaCl}_{2}$ ) (Bell et al., 2011). The aorta was immediately cannulated to the Langendorff perfusion apparatus (Powerlab/8sp, ADInstruments Pty Ltd., Australia) and perfused retrogradely with $\mathrm{KH}$ solution (36.5 $\left.\pm 1^{\circ} \mathrm{C}, \mathrm{pH} 7.35-7.40\right)$ at a constant flow mode. $\mathrm{KH}$ solution was equilibrated with $95 \% \mathrm{O}_{2}$ and $5 \% \mathrm{CO}_{2}$ mixed gas and the perfusion speed was adjusted to $10-15 \mathrm{~mL} / \mathrm{min}$ to maintain the pressure between 70 and $80 \mathrm{mmHg}$. The hearts beat spontaneously at sinus rhythm. Immediately after a stabilization period to stabilize the heart beat and state, experiment was initiated with a 15 min vehicle control period on all acceptable 
heart. After the perfusion of the vehicle control, the equilibrated hearts were perfused with a control $\mathrm{KH}$ solution or a XSN testing solution. In control group, $\mathrm{KH}$ solution was continuously perfused for $10 \mathrm{~min}$; in XSN group, the hearts were perfused with $0.4 \mathrm{~g} / \mathrm{L} \mathrm{XSN}$ for $10 \mathrm{~min}$. XSN was freshly dissolved in $\mathrm{KH}$ solution and filtered with $0.22 \mu \mathrm{m}$ filter before drug application. Finally, all the hearts were perfused with $\mathrm{KH}$ solution for another $10 \mathrm{~min}$. The following parameters were measured: $\mathrm{R}$ wave amplitude, $\mathrm{T}$ wave amplitude, QRS interval, QT interval, and RR interval.

\section{Primary Cardiomyocytes Isolation}

The Wistar rats were anesthetized using isoflurane. Hearts were rapidly excised immediately immersed in cold Krebs-Henseleit (KH) solution described as above and perfused on a Langendorff apparatus for $15 \mathrm{~min}$ with Standard $\mathrm{KH}$ buffer at $37^{\circ} \mathrm{C}$ gassed with $100 \% \mathrm{O}_{2}$, followed by changing to a low $\mathrm{Ca}^{2+} \mathrm{KHB}$ solution (containing $105.1 \mathrm{mM} \mathrm{NaCl}, 3.0 \mathrm{mM} \mathrm{KCl}, 20.0 \mathrm{mM} \mathrm{NaHCO}$, $0.01 \mathrm{mM} \mathrm{CaCl}_{2}, 1.0 \mathrm{mM} \mathrm{KH} \mathrm{PO}_{4}, 1.2 \mathrm{mM} \mathrm{MgSO}, 10 \mathrm{mM}$ glucose, $5.0 \mathrm{mM}$ Na-pyruvate, $10.0 \mathrm{mM}$ taurine, and $5.0 \mathrm{mM}$ mannitol, $\mathrm{pH}$ 7.4) for $10 \mathrm{~min}$ to stop the heartbeat. Then the heart was digested with recirculating low $\mathrm{Ca}^{2+} \mathrm{KHB}$ solution containing $0.08 \mathrm{mg} / \mathrm{mL}$ Liberase TH (Roche-Life Science) at $37^{\circ} \mathrm{C}$ for $50 \mathrm{~min}$. The ventricle was minced and filter into a $50 \mathrm{~mL}$ centrifuge tube with 4-layer gauze, centrifuged twice at $50 \mathrm{~g}$ for 2 min and resuspended with low $\mathrm{Ca}^{2+} \mathrm{KHB}$ solution.

\section{Whole-Cell Patch Clamp Recording}

Action potential in rat ventricular cardiomyocytes were recorded using the current clamp mode of the patch-clamp technique. cardiomyocytes were perfused at room temperature $\left(\sim 22-24^{\circ} \mathrm{C}\right)$ with external solution containing in $\mathrm{mM}$ : $\mathrm{NaCl}, 112 ; \mathrm{NaH}_{2} \mathrm{PO}_{4}$, 1; $\mathrm{NaHCO}_{3}, 24 ; \mathrm{KCl}, 5.4 ; \mathrm{MgCl}_{2}, 1.2 ; \mathrm{CaCl}_{2}, 1.8 ;$ glucose, 10; HEPES, 5; pH 7.4. Borosilicate glass electrodes (Harvard Apparatus, United Kingdom) with a tip resistance of $2 \sim 5 \mathrm{M} \Omega$ were filled with the internal solution containing (in $\mathrm{mM}$ ): $\mathrm{KCl}$, 120; $\mathrm{MgCl}_{2}, 2 ; \mathrm{CaCl}_{2}, 1 ; \mathrm{Na}_{2} \mathrm{ATP}, 3$; EGTA, 11; and HEPES, 10, $\mathrm{pH}$ 7.3. Cardiomyocytes were stimulated by applying an intracellular depolarizing stimulus with an amplitude as 2 times of action potential threshold via a digital pulse generator built in the Axopatch ${ }^{\mathrm{TM}}$ 200B amplifier.

$\mathrm{Na}_{\mathrm{V}} 1.5$ and hERG current was recorded from stably transfected human $\mathrm{Na}_{V} 1.5$ or hERG channel relatively using the voltage-clamp mode of patch clamp techniques (Finkel et al., 2006). The $I_{\mathrm{Na}}$ was activated at -120 to $20 \mathrm{mV}$ sustained for $50 \mathrm{~ms}$ with $5 \mathrm{mV}$ increments at $1 \mathrm{~Hz}$. The transmembrane ion currents and potentials were recorded in the whole-cell recording mode using an Axopatch ${ }^{\mathrm{TM}}$ 200B amplifier (Molecular Devices, LLC, Sunnyvale, CA, United States), and the data were analyzed using pCLAMP 10.3 software (Axon Instruments, Inc.) and Origin 9.1.

\section{Statistical Analysis}

The data values are expressed as the mean \pm standard error and analyzed using a two-tailed paired $t$-test to examine the individual apparent differences. $p<0.05$ was used to indicate a statistically significant difference and $p<0.01$ was used to indicate a highly significant difference.

\section{RESULTS}

\section{Effect of XSN on ECG of Isolated Rat Heart}

Prior the study of the effect of XSN on ion channels we carried out a group of experiment to test the effect of XSN on ECG of isolated Rat hearts with a relatively high concentration of XSN aiming to elicit the maximal effect. Compared with the control, $0.4 \mathrm{~g} / \mathrm{L} \mathrm{XSN}$ induced significant changes in the ECG morphology, including decreased amplitude of $\mathrm{R}$ wave and increased $\mathrm{T}$ wave amplitude (Figure 1 and Table 1). The QRS interval, QT interval and RR interval did not show significant alterations during the perfusion of XSN and upon the 10 min washout of XSN.

\section{Effects of XSN on Peak $\mathrm{Na}^{+}$Current of Rat Primary Cardiomyocytes}

The inhibitive effect of XSN on peak $\mathrm{Na}^{+}$current of rat ventricular cardiomyocytes was carried in the presence of $0.4 \mathrm{~g} / \mathrm{L}$ XSN. As shown in Figure 2, peak $\mathrm{Na}^{+}$current was significantly suppressed by XSN which is corresponding to the suppression of the action potential amplitude.

\section{Effects of XSN on Human hNav1.5 Channel}

The inhibitory effect of XSN on hNaV1.5 channel was shown as Figure 3A. The inhibition effect of XSN on hNa 1.5 channel was dose-dependent with XSN concentration ranging from 0.025 to $1.6 \mathrm{~g} / \mathrm{L}$, with an $\mathrm{IC}_{50}$ of $0.18 \pm 0.02 \mathrm{~g} / \mathrm{L}$ (Figure 3B). Binding state assay showed that XSN may have the tendency to block hNaV1.5 channel at inactivation state, as shown in Figures 3C-E, $\mathrm{V}_{1 / 2}$ in steady activation curve shifted from $-39.29 \pm 1.41 \mathrm{mV}$ (control, $n=8)$ to $-39.90 \pm 1.49 \mathrm{mV}(0.8 \mathrm{~g} / \mathrm{L} \mathrm{XSN}, n=8$, $p>0.05$ v.s. control). $\mathrm{V}_{1 / 2}$ in steady inactivation curve shifted from $-70.96 \pm 0.68 \mathrm{mV}$ (control, $n=8$ ) to $-75.20 \pm 0.63 \mathrm{mV}$ (0.8 g/L XSN, $n=8, p<0.001$ v.s. control). tau in steady recovery curve shifted from $4.92 \pm 1.24 \mathrm{~ms}$ (control, $n=6$ ) to $3.80 \pm 0.72 \mathrm{~ms}(0.8 \mathrm{~g} / \mathrm{L} \mathrm{XSN}, n=8, p>0.05$ v.s. control).

Compared with control condition, $0.8 \mathrm{~g} / \mathrm{L}$ XSN caused significant use-dependent block at stimulation frequencies of $1 \mathrm{~Hz}(p<0.001)$ and $10 \mathrm{~Hz}(p<0.001)$, which was accentuated when $1.6 \mathrm{~g} / \mathrm{L}$ of XSN $(p<0.001)$ was applied (Figure 4$)$.

\section{Effects of XSN on Human hERG Potassium Channel}

The effect of XSN $(0.4 \mathrm{~g} / \mathrm{L})$ on the hERG channel was shown as Figure 5A. XSN dose-dependently blocked hERG channel with an $\mathrm{IC}_{50}=0.34 \pm 0.01 \mathrm{~g} / \mathrm{L}$ (Figure 5B). Steady-state inhibition assay showed that XSN may have the tendency to block hERG channel at both open and inactivation state, as shown in Figures 5C,D. $\mathrm{V}_{1 / 2}$ in steady-state activation curve shifted from $-1.27 \pm 0.28 \mathrm{mV}$ (control, $n=8$ ) to $-23.79 \pm 2.31 \mathrm{mV}$ (0.4 g/L XSN, $n=8, p<0.001$ v.s. control). $\mathrm{V}_{1 / 2}$ in steady inactivation curve shifted from $-59.87 \pm 0.81 \mathrm{mV}$ (control, $n=8$ ) to $-97.56 \pm 2.25 \mathrm{mV}$ ( $0.4 \mathrm{~g} / \mathrm{L} \mathrm{XSN}, n=8, p<0.001$ v.s. control). 


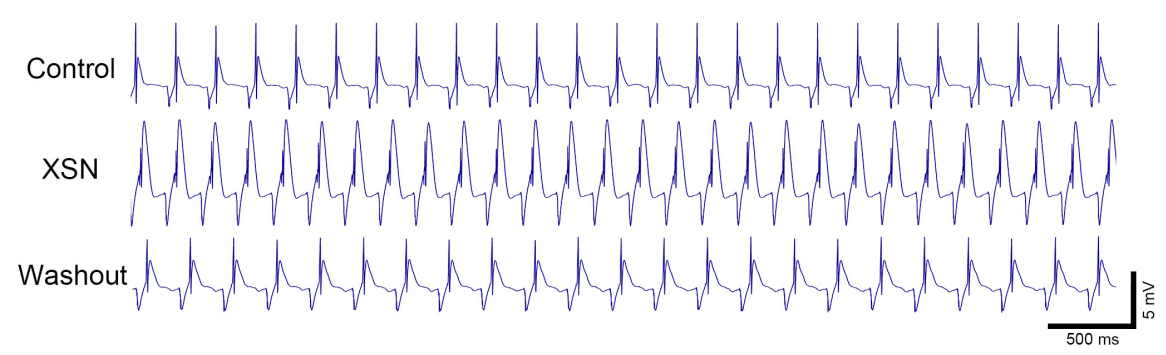

FIGURE 1 | The effect of XSN on the ECG of ex vivo perfused rat heart. XSN at $0.4 \mathrm{~g} / \mathrm{L}$ decreased the R wave amplitude, but increased T wave amplitude without changing QRS and QT interval. Heart rate (RR interval) did not show significant changes during XSN application and at 10 min washout of the medicine.

TABLE 1 | Percentage change of R wave, T wave, QRS, QT, and RR interval (\% of control, $n=5)$.

\begin{tabular}{|c|c|c|c|c|c|}
\hline & R wave & T wave & QRS interval & QT interval & RR interval \\
\hline XSN & $54.00 \pm 5.47^{* * *}$ & $247.71 \pm 41.12^{*}$ & $123.72 \pm 12.26$ & $114.29 \pm 10.73$ & $91.06 \pm 7.06$ \\
\hline Washout & $71.91 \pm 9.93^{*}$ & $103.31 \pm 14.85$ & $111.94 \pm 7.06$ & $116.73 \pm 8.36$ & $104.30 \pm 11.86$ \\
\hline
\end{tabular}

${ }^{*} p<0.05,{ }^{* * *} p<0.001$ vs. control.

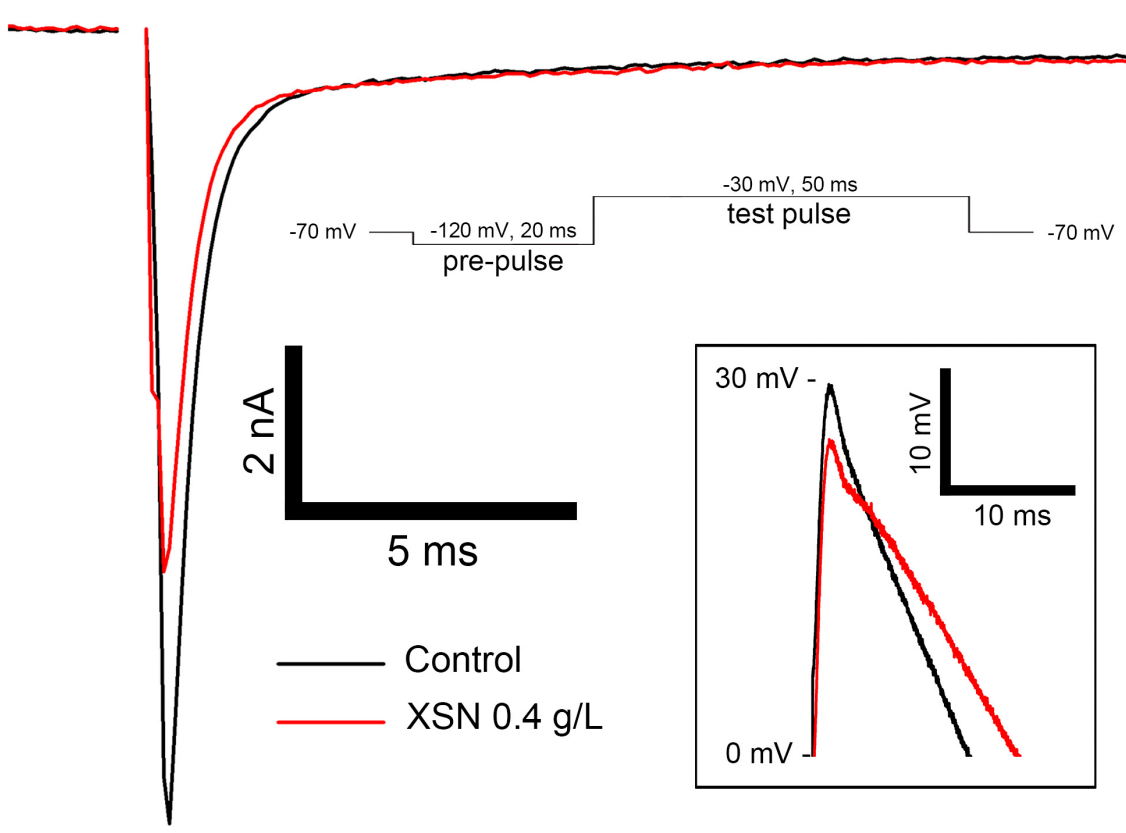

FIGURE 2 | Effect of $0.4 \mathrm{~g} / \mathrm{L}$ XSN on inhibition of peak $\mathrm{Na}^{+}$current on primary rat ventricular myocytes $(n=5)$. The enlarged traces of the peaks of the action potentials are shown in the box on the bottom right.

\section{DISCUSSION}

XSN is a clinically proven antiarrhythmic medicine in China (Yuan and Zhou, 2000). It has been widely used in China for more than 10 years, without sever adverse reactions being reported. It is applicable for moderating premature ventricular contraction (PVC) caused by coronary heart disease and viral myocarditis (Lin et al., 2011). In 2017, the clinical trial of 861 patients was reported in Lancet. The results showed that the mean reduction of PVC by XSN (4604.67 \pm 6990.07$)$ was close to Mexiletine $(4502.86 \pm 5771.70)$ and was significantly higher than placebo group $(1512.47 \pm 8311.14)$. In agreement with the more than 10 years clinical use, there had no pro-arrhythmic phenomenon of XSN being seen in this clinical study. Furthermore, XSN has been reported to treat paroxysmal atrial fibrillation and viral myocarditis (Wang and Lu, 2008; Li and Zhang, 2015). Our previous cellular electrophysiological study showed that XSN displays the property of Class III antiarrhythmic drugs by prolong APD of the cardiac myocytes. In this study, we revealed that XSN blocks hNaV1.5 sodium channel and hERG potassium channel, which has placed XSN as a class I and III antiarrhythmic medicine as the widely used antiarrhythmic drug amiodarone. 


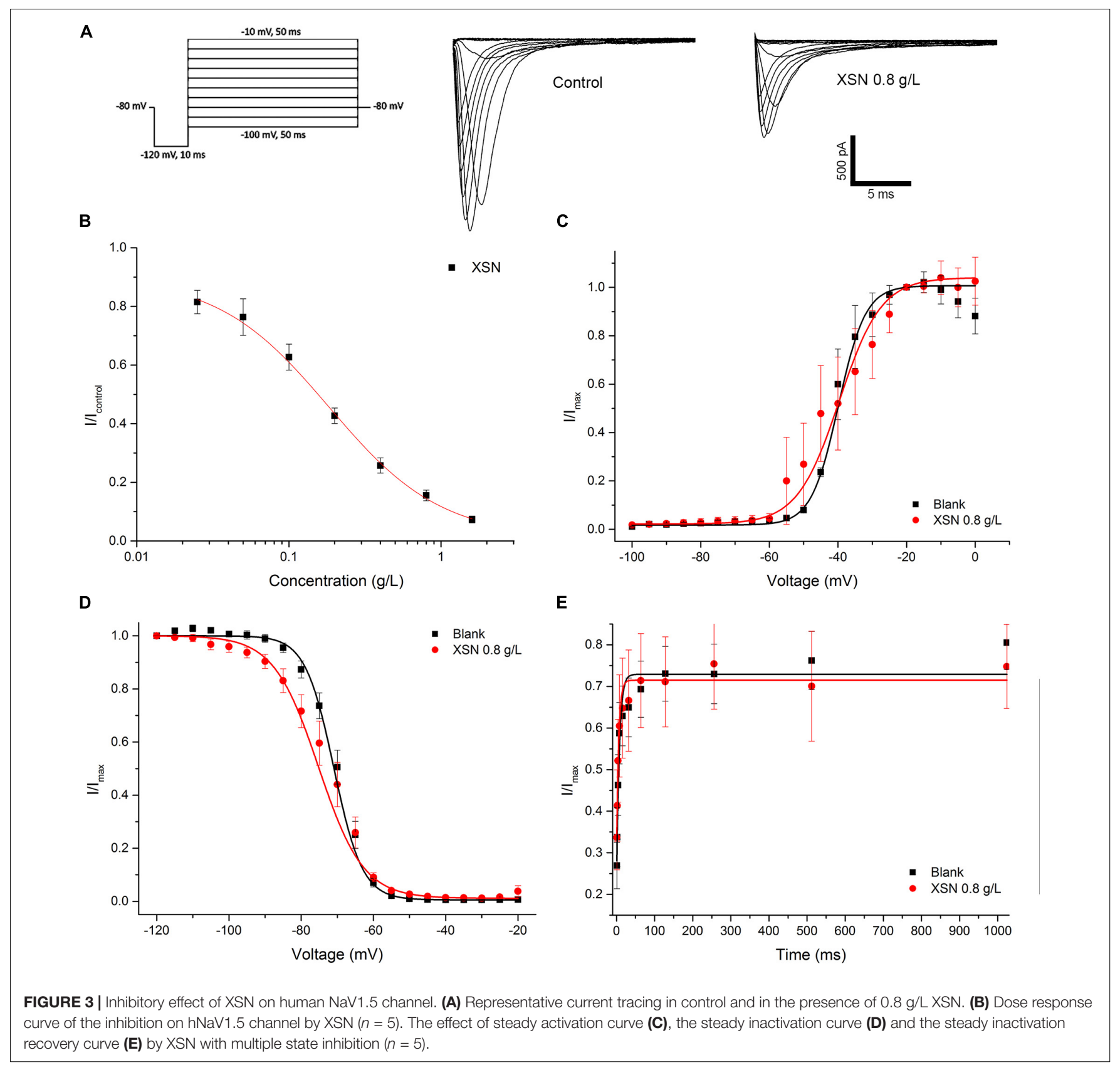

Our results presented here furthered our understanding of the clinical anti-arrhythmic action of XSN: (1) in the ex vivo ECG study on isolated wistar rat heart, the $\mathrm{R}$ wave and $\mathrm{T}$ wave of the ECG showed significant morphological changes after the application of XSN (Figure 1). It is well known, the QRS complex, especially the $\mathrm{R}$ wave amplitude, represents ventricular depolarization caused by transiently activation of sodium channels. The suppression of $\mathrm{R}$ wave suggests the inhibition of fast-voltage-gated sodium channel, which is the anti-arrhythmic mechanism of the Class I anti-arrhythmic agents. In fact, the clinical dose of XSN in human blood serum was much lower than $0.4 \mathrm{~g} / \mathrm{L}$, even though, at this concentration, XSN did not induce Torsade de Pointes (TdP) or other kind of arrhythmia in the isolated rat hearts, which may well be the beneficial outcome of the multicomponent action of XSN.

The cellular electrophysiological assay showed that XSN prolonged $\mathrm{APD}_{90}$ on rat cardiomyocytes (Ma et al., 2017) and reduced the APA as shown in Figure 2, which indicated that XSN may block sodium and potassium channels. These indications were supported by our results shown in Figures 2, 3.

The inhibitory effect of XSN on human Nav1.5 channel shown in Figure 3 displays a dose dependent manner at concentrations ranging from 0.025 to $1.6 \mathrm{~g} / \mathrm{L}$ $\mathrm{XSN}$, and that the human $\mathrm{Na}_{\mathrm{V}} 1.5$ channel was blocked at inactivation state as shown in Figure 3. As peak sodium and late sodium current are produced by Nav1.5 


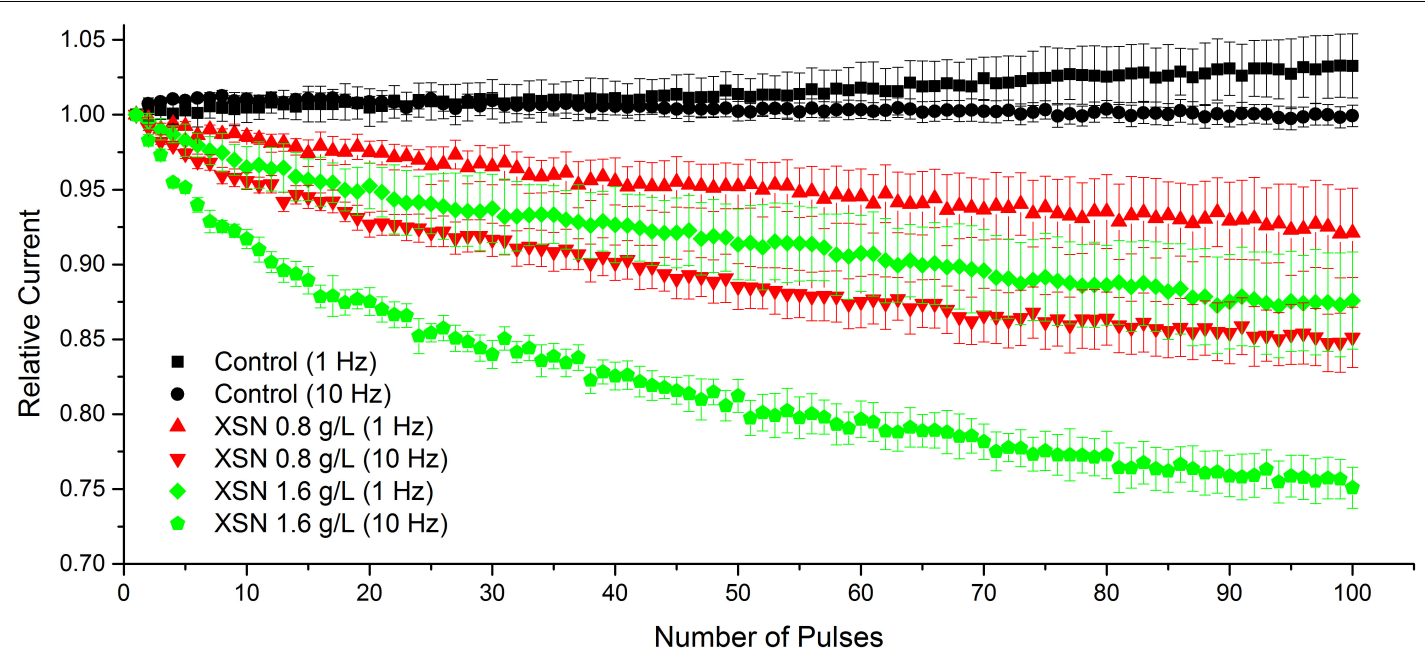

FIGURE 4 | Use-dependent block of $\mathrm{I}_{\mathrm{Na}}$ following acceleration from 1 to $10 \mathrm{~Hz}$ in control and after exposure to 0.8 and $1.6 \mathrm{~g} / \mathrm{L}$ XSN. For control $n=12,1 \mathrm{~Hz} n=7$, $10 \mathrm{~Hz} n=5$. Both of 0.8 and $1.6 \mathrm{~g} / \mathrm{L} \mathrm{XSN}$ in the frequency of 1 or $10 \mathrm{~Hz}$ showed significantly use-dependently block of Nav1.5 channel.

\section{A}
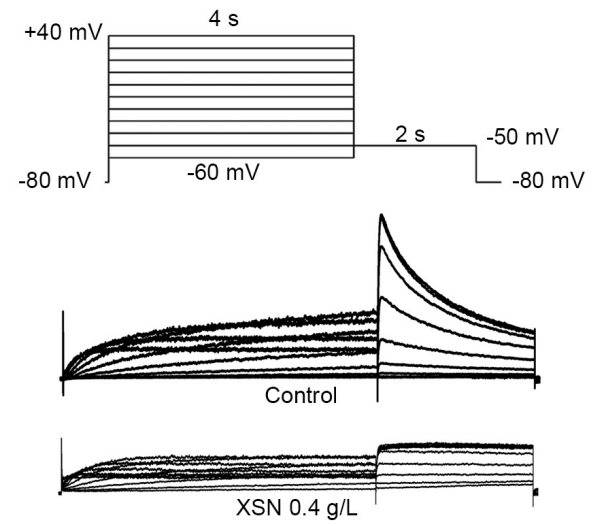

C

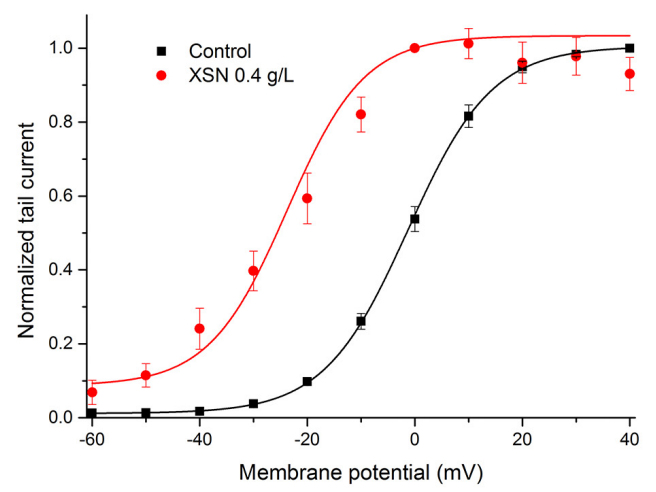

B
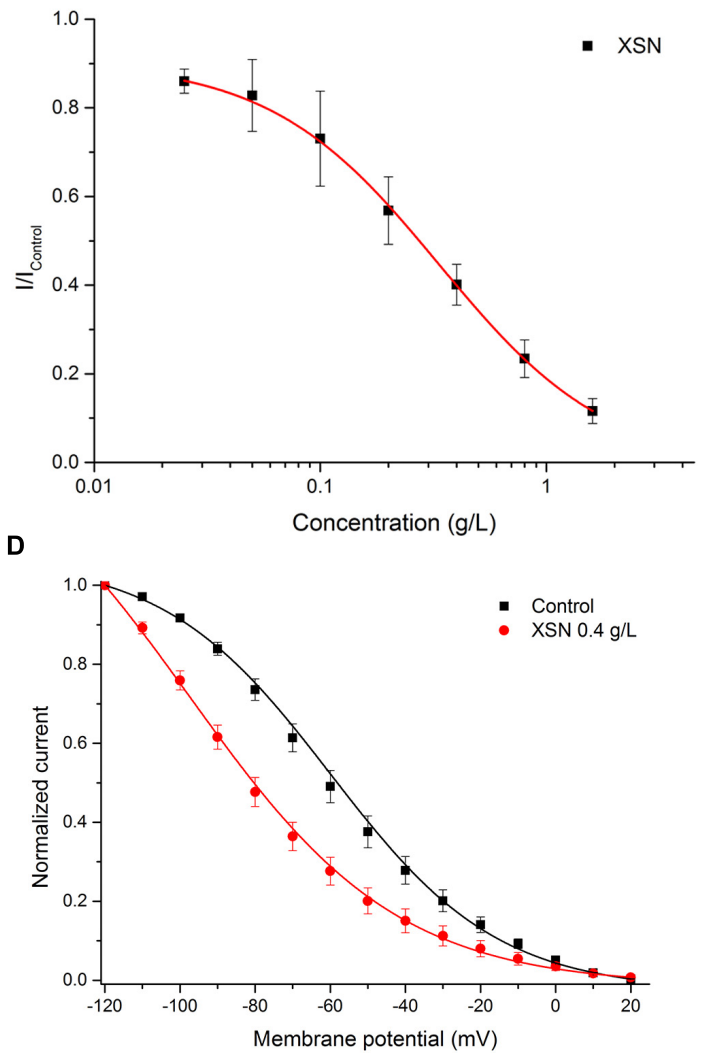

FIGURE 5 | Inhibitory effect of XSN on hERG channel. (A) Representative current tracing in control and in the presence of $0.4 \mathrm{~g} / \mathrm{L}$ XSN. (B) Dose response curve of the inhibition on hERG channel by XSN $(n=5)$. The effect of steady activation curve (C), the steady inactivation curve (D) by XSN with multiple state inhibition $(n=8)$.

channel (Maltsev et al., 2009), the inactivation blockage of $\mathrm{Na}_{\mathrm{V}} 1.5$ suggests the possibility of a post-repolarization refractoriness period, which provide mechanistic explanation for XSN's inhibition of the premature hearts beat in patients. Additionally, $0.8 \mathrm{~g} / \mathrm{L} \mathrm{XSN}$ caused significant usedependent block at both frequencies tested (Figure 4): $1 \mathrm{~Hz}(p<0.001)$ and $10 \mathrm{~Hz}(p<0.001)$, which helps to explain the clinical therapeutic effect of XSN on tachycardia 
(Hondeghem and Katzung, 1977; Campbell, 1983; Wang et al., 2015; Potet et al., 2016).

XSN blocks hERG potassium channel which would prolong APD and increase the effective refractory period of the heart which in turns inhibits PVC.

PVCs are a relatively common occurrence caused by irritated ectopic foci in the cardiac ventricle (Adams et al., 2012), which are responsible for considerable morbidity and mortality (Takemoto et al., 2005). It may cause hemodynamic deterioration and reversible left ventricular dysfunction and can act as electrophysiological markers to other cardiac diseases such as cardiomyopathy and ischemic heart disease (Zaborska et al., 2006; Kanei et al., 2008). It has been demonstrated that the quantitative additional mortality risk on exercise test presented by frequent PVCs is similar to ischemia (Iacovino, 2001). Clinical presentation of PVCs may be quite variable, ranging from an incidental finding on electrocardiogram (ECG) to congestive heart failure (Morganroth, 1984; Santoro et al., 2014). For patients with symptoms that can be attributed to PVCs. the drug therapy of beta blockers or class I or III antiarrhythmic agents is effective and recommended (Zipes et al., 1984; Capucci et al., 1991; Zou et al., 2011; Zhong et al., 2014).

$\mathrm{XSN}$, as a multicomponent medicine, inhibit PVC, ischemia induced cardiac arrhythmia and virus myocarditis, which show an abroad spectrum pharmacological actions. As we know that arrhythmias more often is induced by other underlying cardiac conditions, therefore it is a multifactor disease which would need multicomponent medicine to treat. XSN has displayed class I and III antiarrhythmic properties, but did not induce arrhythmia at very high concentration, we are aiming to further our study to discover how the multicomponent in XSN coordinated to achieve the therapeutic efficacy without causing adverse reactions.

\section{CONCLUSION}

Our results presented here have proved further that multiherbal antiarrhythmic medicine XSN displays clear cellular electrophysiological mechanism that support its clinical efficacy in patients, and the results also assemble the properties processed by antiarrhythmic chemical drugs although the data present the overall effect of a multicomponent medicine. We therefore conclude that: (1) XSN is a class I and III antiarrhythmic multicomponent medicine; (2) The dosedependent and completely reversible effect of XSN indicate a low toxicity property of XSN; (3) The discoveries that XSN blocks hNav1.5 and hERG backed by the PAP suppression and prolongation of APD are the cellular electrophysiological

\section{REFERENCES}

Adams, J. C., Srivathsan, K., and Shen, W. K. (2012). Advances in management of premature ventricular contractions. J. Interv. Card. Electrophysiol. 35, 137-149. doi: 10.1007/s10840-012-9698-x

Bell, R. M., Mocanu, M. M., and Yellon, D. M. (2011). Retrograde heart perfusion: the langendorff technique of isolated heart perfusion. J. Mol. Cell. Cardiol. 50, 940-950. doi: 10.1016/j.yjmcc.2011.02.018 mechanism that support the clinical therapeutic effect of XSN in treating PVC and tachyarrhythmia in patients.

\section{STUDY LIMITATIONS}

Although our results have clearly placed XSN into the groups of class I and III antiarrhythmic drugs, the effect of XSN in animal arrhythmic models is lacking in this report. Since there is strong evidence that XSN is a clinically effective antiarrhythmic medicine in treating PVC and ischemia induced cardiac arrhythmia, it would add valuable laboratory research data if we study the effect of XSN in animal ischemia and arrhythmia models. We are aiming in our future report to demonstrate the in vivo antiarrhythmic effect of XSN.

\section{AUTHOR CONTRIBUTIONS}

Y-lM conceived and designed the experiments. TW, WX, and JY performed the experiments. TW and Y-lM analyzed the data. YZ and Y-lM contributed reagents, materials, and analysis tools. TW and Y-lM wrote the paper. CE and RW discussed the experimental design.

\section{FUNDING}

This work was supported by the Chinese Medicine Research Fund in University of Oxford. The grant was sponsored by Shaanxi Momentum Pharmaceutical Co., Ltd. Shaanxi Momentum Pharmaceutical Co., Ltd. had no involvement in the study design, data collection and analysis, decision to publish, or preparation of the manuscript.

\section{ACKNOWLEDGMENTS}

We thank YZ for providing the stable transfected cell lines and Prof. Carolyn Carr for stimulating discussions, equipment, and lab space supports.

\section{SUPPLEMENTARY MATERIAL}

The Supplementary Material for this article can be found online at: https://www.frontiersin.org/articles/10.3389/fphar. 2019.00070/full\#supplementary-material

Burashnikov, A., Petroski, A., Hu, D., Barajas-Martinez, H., and Antzelevitch, C. (2012). Atrial-selective inhibition of sodium-channel current by wenxin keli is effective in suppressing atrial fibrillation. Heart Rhythm 9, 125-131. doi: 10.1016/j.hrthm.2011.08.027

Campbell, T. J. (1983). Kinetics of onset of rate-dependent effects of class I antiarrhythmic drugs are important in determining their effects on refractoriness in guinea-pig ventricle, and provide a theoretical basis for their subclassification. Cardiovasc. Res. 17, 344-352. doi: 10.1093/cvr/17.6.344 
Capucci, A., Di, G. P., Boriani, G., Carini, G., Balducelli, M., Frabetti, L., et al. (1991). A double-blind crossover comparison of flecainide and slow-release mexiletine in the treatment of stable premature ventricular complexes. Int. J. Clin. Pharmacol. Res. 11, 23-33.

Finkel, A., Wittel, A., Yang, N., Handran, S., Hughes, J., and Costantin, J. (2006). Population patch clamp improves data consistency and success rates in the measurement of ionic currents. J. Biomol. Screen. 11, 488-496. doi: 10.1177/ 1087057106288050

Frommeyer, G., and Eckardt, L. (2016). Drug-induced proarrhythmia: risk factors and electrophysiological mechanisms. Nat. Rev. Cardiol. 13, 36-47. doi: 10. 1038/nrcardio. 2015.110

Guo, R., Zhang, X., Su, J., Xu, H., Zhang, Y., Zhang, F., et al. (2018). Identifying potential quality markers of Xin-Su-Ning capsules acting on arrhythmia by integrating UHPLC-LTQ-Orbitrap, ADME prediction and network target analysis. Phytomedicine 44, 117-128. doi: 10.1016/j.phymed.2018.01.019

Hondeghem, L. M., and Katzung, B. G. (1977). Time-and voltage-dependent interactions of antiarrhythmic drugs with cardiac sodium channels. Biochim. Biophys. Acta Rev. Biomembr. 472, 373-398. doi: 10.1016/0304-4157(77)90 003-X

Hua, W., Gao, R.-L., Zhao, B.-C., Wang, J., Chen, X.-H., Cai, C., et al. (2015). The efficacy and safety of Wenxin Keli in patients with frequent premature ventricular contractions: a randomized, double-blind, placebo-controlled, parallel-group, multicenter trial. Chin. Med. J. 128, 2557-2564. doi: 10.4103/ 0366-6999.166026

Iacovino, J. R. (2001). Comparison of the mortality between ischemia and frequent premature ventricular contractions on an exercise test. J. Insur. Med. 33, $222-226$.

Kanei, Y., Friedman, M., Ogawa, N., Hanon, S., Lam, P., and Schweitzer, P. (2008). Frequent premature ventricular complexes originating from the right ventricular outflow tract are associated with left ventricular dysfunction. Ann. Noninvasive Electrocardiol. 13, 81-85. doi: 10.1111/j.1542-474X.2007.00204.x

Li, F., and Zhang, C. (2015). Clinical study of xinsuning capsule in the treatment of paroxysmal atrial fibrillation. Med. Front. 5, 95-97.

Li, N., Ma, K.-J., Wu, X.-F., Sun, Q., Zhang, Y.-H., and Pu, J.-L. (2007). Effects of chinese herbs on multiple ion channels in isolated ventricular myocytes. Chin. Med. J. 120, 1068-1074. doi: 10.1097/00029330-200706020-00008

Lin, L., Liu, Y., and Lin, H. (2011). Observation on twenty-six cases of frequent premature ventricular contractions treated with xinsuning capsules. People's Mil. Surg. 54:512.

Ma, Y., Wang, T., Ellory, C., Wilkins, R., Carr, C., Mao, P., et al. (2017). "Investigation of the active antiarrhythmic components of the multi-herbal medicine Xin Su Ning," in Proceedings Of The British Pharmacological Society, (Oxford: Oxford University Research Archive).

Ma, Y.-L., Bates, S., and Gurney, A. M. (2006). The effects of paeonol on the electrophysiological properties of cardiac ventricular myocytes. Eur. J. Pharmacol. 545, 87-92. doi: 10.1016/j.ejphar.2006.06.064

Maltsev, V. A., Kyle, J. W., and Undrovinas, A. (2009). Late Na+ current produced by human cardiac $\mathrm{Na}+$ channel isoform Nav1.5 Is Modulated By Its Beta1 subunit. J. Physiol. Sci. 59, 217-225. doi: 10.1007/s12576-009-0029-7

Morganroth, J. (1984). Premature ventricular complexes: diagnosis and indications for therapy. JAMA 252, 673-676. doi: 10.1001/jama.1984.03350050061030

O'rourke, B., Liu, T., and Foster, D. B. (2016). Seeing the forest for the trees. Circ. Res. 119, 1170-1172. doi: 10.1161/CIRCRESAHA.116.310066

Potet, F., Vanoye, C. G., and George, A. L. (2016). Use-dependent block of human cardiac sodium channels by GS967. Mol. Pharmacol. 90, 52-60. doi: 10.1124/ mol.116.103358

Rosen, M. R. (1988). Mechanisms for arrhythmias. Am. J. Cardiol. 61, A2-A8. doi: 10.1016/0002-9149(88)90735-7

Santoro, F., Biase, L. D., Hranitzky, P., Sanchez, J. E., Santangeli, P., Perini, A. P., et al. (2014). Ventricular fibrillation triggered by PVCs from papillary muscles: clinical features and ablation. J. Cardiovasc. Electrophysiol. 25, 1158-1164. doi: $10.1111 /$ jce. 12478
Sun, Y., Liu, Y., and Chen, K. (2016). Roles and mechanisms of ginsenoside in cardiovascular diseases: progress and perspectives. Sci. China Life Sci. 59, 292-298. doi: 10.1007/s11427-016-5007-8

Takemoto, M., Yoshimura, H., Ohba, Y., Matsumoto, Y., Yamamoto, U., Mohri, M., et al. (2005). Radiofrequency catheter ablation of premature ventricular complexes from right ventricular outflow tract improves left ventricular dilation and clinical status in patients without structural heart disease. J. Am. Coll. Cardiol. 45, 1259-1265. doi: 10.1016/j.jacc.2004. 12.073

Tian, G., Sun, Y., Liu, S., Li, C., Chen, S., Qiu, R., et al. (2018). Therapeutic effects of wenxin keli in cardiovascular diseases: an experimental and mechanism overview. Front. Pharmacol. 9:1005. doi: 10.3389/fphar.2018.01005

Wang, C., and Lu, J. (2008). Observation on thirty cases of viralmyocarditis treated with xinsuning capsules. Shanxi Zhongyi 29:1362. doi: 10.3969/j.issn.1000-7369. 2008.10.068

Wang, X., Wang, Y., Feng, X., Lu, Y., Zhang, Y., Wang, W., et al. (2016). Systematic review and meta-analysis of randomized controlled trials on wenxin keli. Drug Des. Dev. Ther. 10, 3725-3736. doi: 10.2147/DDDT.S112333

Wang, Y., Mi, J., Lu, K., Lu, Y., and Wang, K. (2015). Comparison of gating properties and use-dependent block of Nav1. 5 and Nav1. 7 channels by antiarrhythmics mexiletine and lidocaine. PLoS One 10:e0128653. doi: 10.1371/ journal.pone.0128653

Weiss, J. N., Garfinkel, A., Karagueuzian, H. S., Nguyen, T. P., Olcese, R., Chen, P. S., et al. (2015). Perspective: a dynamics-based classification of ventricular arrhythmias. J. Mol. Cell. Cardiol. 82, 136-152. doi: 10.1016/j.yjmcc.2015. 02.017

Williams, E. V. (1992). Classifying antiarrhythmic actions: by facts or speculation. J. Clin. Pharmacol. 32, 964-977. doi: 10.1002/j.1552-4604.1992.tb03 797.x

Yuan, S., and Zhou, C. (2000). Observation on sixty cases of rapid arrhythmia treated with xinsuning capsules. J. Shandong Univ. TCM 24, 290-293. doi: 10.3969/j.issn.1007-659X.2000.04.027

Zaborska, B., Stec, S., Flasińska, K., Piluś, A., and Kułakowski, P. (2006). Echocardiography and tissue Doppler imaging in assessment of haemodynamics in patients with idiopathic, premature ventricular complexes. Polski Merkuriusz Lekarski: Organ Polskiego Towarzystwa Lekarskiego 20, 302-304.

Zhai, J., Yin, X., Yang, X., and Zhang, J. (2017). Xinsuning capsule for the treatment of premature ventricualr contraction: a multicenter randomised clinical trial. Lancet 390:S61. doi: 10.1016/S0140-6736(17)33199-9

Zhong, L., Lee, Y.-H., Huang, X.-M., Asirvatham, S. J., Shen, W.-K., Friedman, P. A., et al. (2014). Relative efficacy of catheter ablation vs antiarrhythmic drugs in treating premature ventricular contractions: a single-center retrospective study. Heart Rhythm 11, 187-193. doi: 10.1016/j.hrthm.2013.10.033

Zipes, D. P., Prystowsky, E. N., and Heger, J. J. (1984). Amiodarone: electrophysiologic actions, pharmacokinetics and clinical effects. J. Am. Coll. Cardiol. 3, 1059-1071. doi: 10.1016/S0735-1097(84)80367-8

Zou, J., Zhang, J., Jia, Z., and Cao, K. (2011). Evaluation of the traditional chinese medicine shensongyangxin capsule on treating premature ventricular contractions: a randomized, double-blind, controlled multicenter trial. Chin. Med. J. 124, 76-83.

Conflict of Interest Statement: The authors declare that the research was conducted in the absence of any commercial or financial relationships that could be construed as a potential conflict of interest.

Copyright (C) 2019 Wang, Xie, Yu, Ellory, Wilkins, Zhu and Ma. This is an open-access article distributed under the terms of the Creative Commons Attribution License (CC BY). The use, distribution or reproduction in other forums is permitted, provided the original author(s) and the copyright owner(s) are credited and that the original publication in this journal is cited, in accordance with accepted academic practice. No use, distribution or reproduction is permitted which does not comply with these terms. 